\title{
Survey on IOT based Home Automation
}

\author{
Roshni Bhandari \\ Assistant professor \\ Computer department \\ S.S.Agrawal Institute of \\ Engineering and Technology \\ Navsari, India
}

\author{
Patel Darshan M. \\ Student \\ Computer Engineering \\ S.S.Agrawal Institute of \\ Engineering and Technology \\ Navsari, India
}

\author{
Patel Keyur D. \\ Student \\ Computer Engineering \\ S.S.Agrawal Institute of \\ Engineering and Technology \\ Navsari, India
}

\author{
Dhimar Richi K. \\ Student \\ Computer Engineering \\ S.S.Agrawal Institute of Engineering and Technology \\ Navsari, India
}

\begin{abstract}
IOT is now a days most preferred technology. A system of interrelated computing devices, mechanical and digital machines, objects, animals or people that are provided with unique identifiers (UIDs) and the ability to transfer data over a network without requiring human-to-human or human-tocomputer interaction. One of the major sides of IOT is a smart home. Smart home is very helpful for the peoples to make life easy. Home Automation is the automatic or semiautomatic control and monitoring of household appliances and residential house features like doors, Gate and even the windows. The IOT definition has been evolved due to convergence of multiple technologies like, The Real Time Analysis, Machine Learning, Commodities Sensors and Embedded systems. The IOT technology is most synonyms with products pretending to the concept of "Smart Home" in the consumer market. Home Automation is important because it is time saving as the lights and fan can be easily turned on and off without wasting much time by reaching to the switch and turn on the light. The doors can also be opened without opening manually the user can opened by sitting on chair. The water level indicator is used for water indicator from the water tank. In that, whenever the water gets low there will be sensor which will start the water motor automatically and when the water tank gets full, again the water motor will get off automatically. For the fire safety, the fire sensor will be present at home which will detect the fire or the smoke and alert the house members. The control system will be under the users mobile phone where there will be application.
\end{abstract}

\section{Keywords}

Home automation, Arduino UNO, sensors, Bluetooth module, WIFI module, Speech recognition, smart phone, Android application.

\section{INTRODUCTION}

The internet of things, or IOT, is a system of interrelated computing devices, mechanical and digital machines, objects, animals or people that are provided with unique identifiers (UIDs) and the ability to transfer data over a network without requiring human-to-human or human-to-computer interaction. One of the major sides of IOT is a smart home. As we are in the era of never-ending growth of the internet and its application, smart home system or home automation system is highly increasing to provide comfort in life and improving the quality of life.
In this paper, we present an IOT based low-cost smart home automation system. The main objective of this system is to make human life easy and comfortable by using IOT. Now a day as people are so busy with their work pressure so the will be looking for a smarter life style.

The definition of the Internet of things has evolved due to the convergence of multiple technologies, realtime analytics, machine learning, commodity sensors, and embedded systems. Traditional fields of embedded systems, control systems, automation (including home) and others all contribute to enabling the Internet of Things. In the consumer market, IoT technology is most synonymous with products pertaining to the concept of the "smart home", covering devices and appliances (such as lighting fixtures, thermostats, home security systems and cameras, and other home appliances) that support one or more common ecosystems, and can be controlled via devices associated with that ecosystem, such as Smartphone's and smart speakers. Long-term benefits could include energy savings by automatically ensuring lights and electronics are turned off. A smart home or automated home could be based on a platform or hubs that control smart devices and appliances.

Home automation or domotics is building automation for a home, called a smart home or smart house. A home automation system will control lighting, climate, entertainment systems, and appliances. It may also include home security such as access control and alarm systems. A home automation system typically connects controlled devices to a central hub.

The form of home automation focuses on making it possible for older adults and people with disabilities to remain at home, safe and comfortable. Home automation is becoming a viable option for older adults and people with disabilities who would prefer to stay in the comfort of their homes rather than move to a healthcare facility. This field uses much of the same technology and equipment as home automation for security, entertainment, and energy conservation but tailors it towards older adults and people with disabilities ${ }^{[1]}$.

\section{METHODOLOGY}

\subsection{Bluetooth Module ${ }^{[2]}$}

Bluetooth module is used to connect the Arduino with the Smartphone and using the phone user can control the things connect to the Arduino. Due the module the user can control the things by sitting away from that thing. 


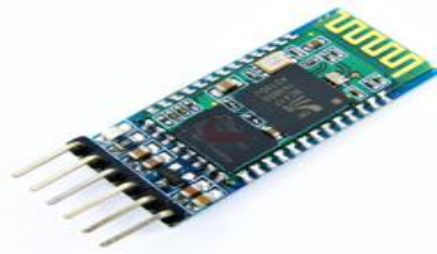

Fig 1: Bluetooth Module[3]

Above figure is Bluetooth module to be used for connectivity with the mobile device. As the Bluetooth connection is wireless the user can control but connecting it and operates through the mobile phone using the application.

\subsection{Arduino UNO}

Arduino UNO is an open source platform which consist of both , a physical programmable circuit board(also called as a microcontroller) and the software that runs on your computer, used to write and upload computer code to the physical board. The board is equipped with sets of digital and analog input/output (I/O) pins that may be interfaced to various expansion boards (shields) and other circuits.

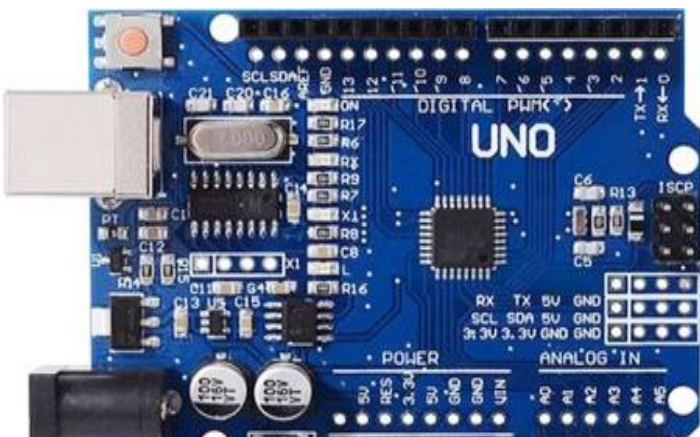

Fig 2: Arduino UNO[4]

\subsection{Android application}

An Android application is a software application running on the Android platform. Because the Android platform is built for mobile devices, Android app is designed for a Smartphone or a tablet running on the Android OS. An Android application is interfaced in two different interfaces for using Home Automation by using Bluetooth communication protocol.

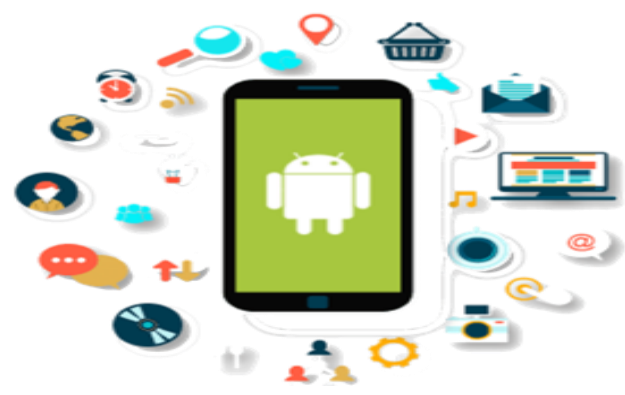

Fig.3 Applications[5]

The two different types of interface for the users are as under:

\subsubsection{Speech Recognition Interface:}

Speech recognition is the ability of a machine or program to identify words and phrases in spoken language and convert to machine language. In this interface, the user will get the facility to take the control by speaking only or in other words user gives command to do the work. The user speaks and the particular function is called and the Arduino takes the command and then the work is completed.

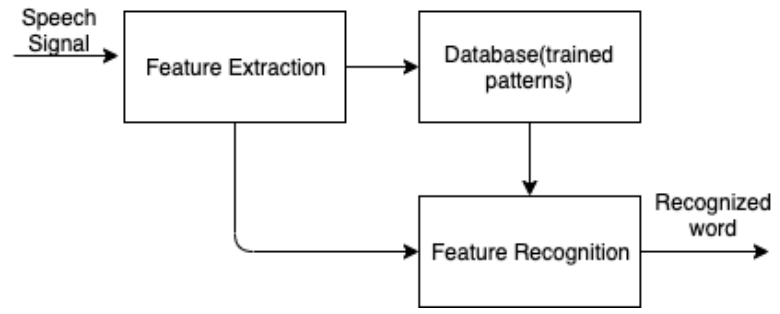

Fig 4: Speech Recognition[6]

The application has this feature because the people who would use it is not fixed so anybody will use it so some can speak in proper English and for this feature positive side is that when the person is not able operate the mobile phone than that person can use this feature.

\subsubsection{Tabs Interface:}

In this the application has feature of speech as well as control using the tabs means by pressing the buttons according to the function. In this, the tabs are given particular function for operations and also have symbols on it so that the user can easily get idea of the button.

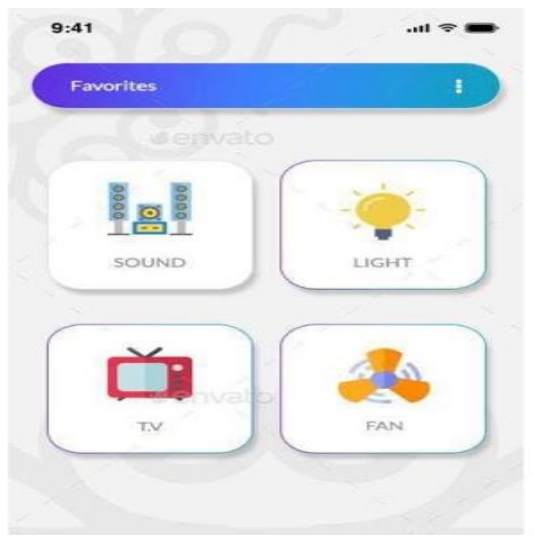

Fig 5: Buttons[7]

\subsection{Zig Bee ${ }^{[8]}$}

ZigBee is an IEEE 802.15 standard used in home automation technology and very closely resembles Bluetooth and Wi-Fi standards. Zigbee devices are attractive largely because of its low power consumption and open specifications which makes the devices ideal for battery operated uses. Zigbee, like ZWave is a mesh protocol, where devices can talk to one another, and can act as repeaters. Even though with so many advantages, the technology has not gained a large market share, mainly because of the incompatibility of devices among many different vendors.

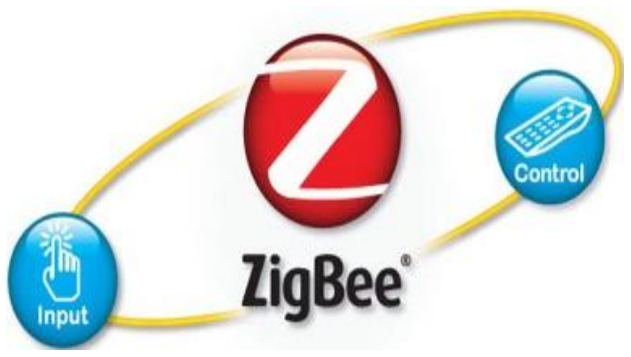

Fig 6: Zigbee[9] 
However, Zigbee proves useful in research, with many universities developing devices such as TelosB, from University of California Berkeley that can be used as part of a wireless sensor network to monitor environmental conditions. TelosB motes are relatively inexpensive, can be used as a transmitter and a receiver and useful for inexpensive custom sensors.

\subsection{Z-wave ${ }^{[8]}$}

Z-Wave is the most widely used technology in home automation systems, and by far the most widely accepted technology. It offers good network reliability and stability. ZWave is one of the oldest available home automation protocols.

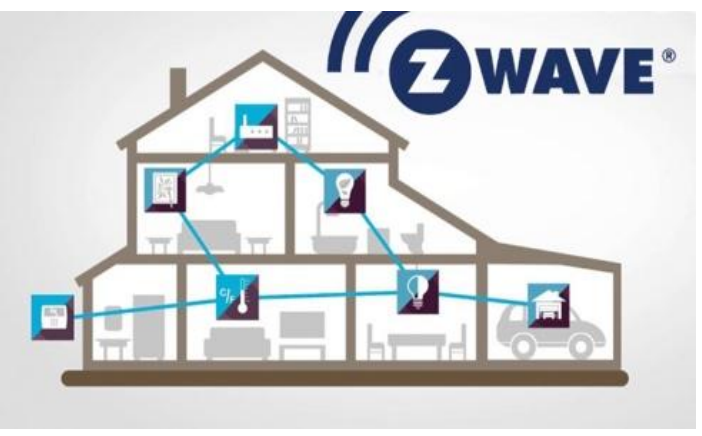

Fig 7: Z-wave[10]

The best feature of Z-Wave devices is their crosscompatibility among different branded systems. Each ZWave device has a unique network ID and each network has a unique identification thus making the system secure. Z- Wave is a mesh protocol, and thus the devices can talk to one another. Z-Wave operating frequency varies with the region; the frequency is $908.42 \mathrm{MHz}$ in the US and $868.42 \mathrm{MHz}$ in Europe. Also, the signal range offered by Z-Wave is high, in the range of 30 meters, and it is possible to extend the range of devices by using them as repeaters. As the signal passes from one device to another, it gains a range of another 30 meters. This process is called hopping, and it can be done to extend the signal using a maximum of 4 devices. However, beyond 4 devices, the $\mathrm{Z}$-Wave protocol terminates the signal.

\section{$2.6 \times 10^{[8]}$}

$\mathrm{X} 10$ is one of the oldest available home automation standards. The technology is still in the market despite tough competition from newer standards. There are reportedly 10 million X10 devices in US alone. An advantage of X10 is that it can use either wired power line or wireless radio communication methods.

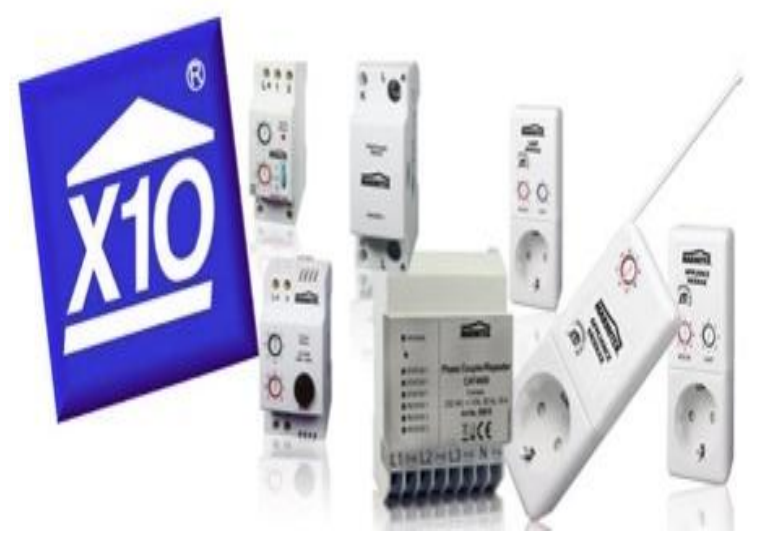

Fig 8: X-10[11]
However, the transmission of messages occur one command at a time. This is one of the biggest disadvantages of X10 because multiple, concurrent X10 signals may lead to decoding issues resulting lost commands. Nevertheless, X10 is inexpensive and many devices are available.

\subsection{Wi-fi ${ }^{[8]}$}

Wi-Fi is another wireless technology that is used to connect home automation appliances based on the IEEE 802.11 standards. It operates in $2.4 \mathrm{GHz}$ and $5 \mathrm{GHz}$ bands which is available worldwide. In home automation, Wi-Fi is mostly used for wireless monitoring and management of home appliances such as power outlets, door, surveillance, and alarms.

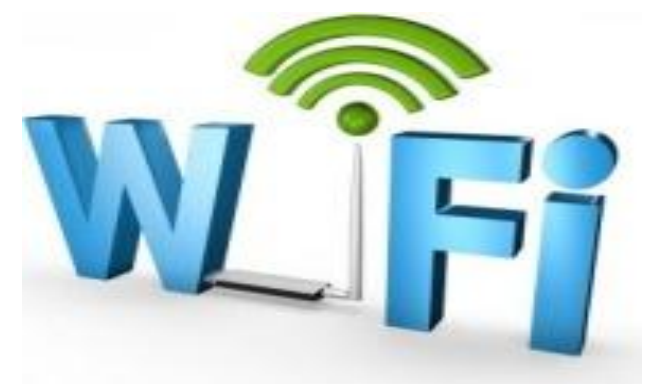

Fig 9: WIFI[12]

\section{LITERATURE SURVEY}

In developed and developing countries the more and more technologies are arriving every year. And of the most important is the IOT. IOT can be used in many sectors of the technology. IOT is also used in home automation which helps to make life easy as well as it is time saving.

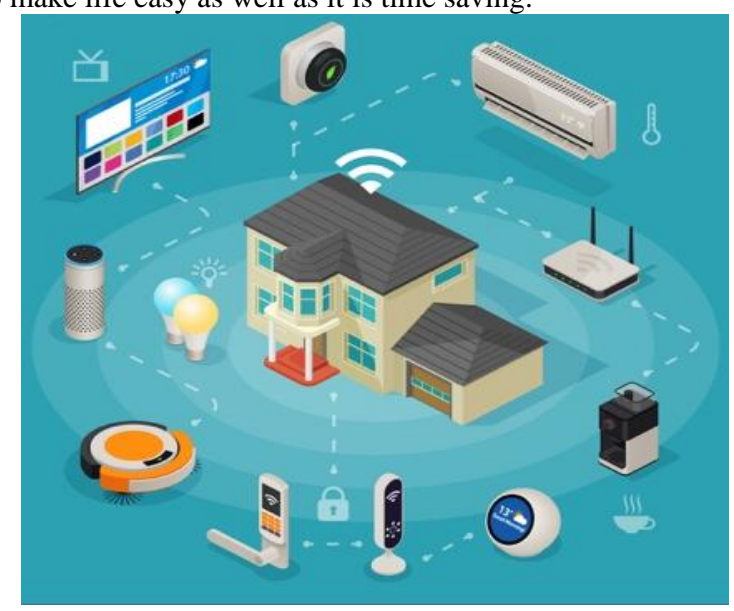

Fig 10: Smart Home[13]

From paper[14], smartphone application nowadays can be utilize for the IOT communication protocols. Application can be used to monitor and control local switches via mobile phone. Many systems are reported in the literature based on single monitoring and controlling mode utilizing text, voice or gesture commands. The system has two different operation modes. The first mode make use of a mobile app interface with virtual switches and slider to monitor and control appliances. The second is chat-based that use text or audio command filter with natural language processing to monitor and control the home appliances. The proposed system is scalable in that it is able to add and remove rooms on demand.

From paper[8], Most of the home automation wireless technologies on the internet of things are based on ZigBee, Z- 
Wave, Wi-Fi, and Bluetooth wireless technologies. While these solutions are good enough, users of smart homes are facing challenges of selecting the best technology.

From paper[15],Our life is becoming more comfortable and smarter with the help of rapid upgrade of technology. Internet of things (IoT) is playing a massive role in this. One of the major sides of IoT is a smart home. As we are in the era of never-ending growth of the internet and its application, smart home system or home automation system is highly increasing to provide comfort in life and improving the quality of life. Now a day as people are so busy with their work pressure so the will be looking for a smarter life style.

From paper[16], The smart mobile terminal operator platform Android is getting popular all over the world with its wide variety of applications and very large use in our daily life. Considering the fact of increasing demand of home security and automation. The mobile to security system or home automation system interface is established through Bluetooth.

\section{CASE STUDY}

From USA, according to the survey the oldest home automation technology is the X-10 technology. In 1975, the first Smart Home Automation Network Technology, X10 was developed. By 1978, X10 products included a 16 channel command console, a lamp module, and an Home appliance module. After sometime, the wall switch module and the first X10 timer were developed. By 2012, in the United States, according to ABI Research, 1.5 million home automation systems were installed. As per research firm Statista more than 45 million smart home devices will be installed in U.S. homes by the end of the year $2018^{[17]}$.

From China, according to the survey the Tmall Genie is a smart speaker developed by Chinese e-commerce company Alibaba Group, using the intelligent personal assistant service AliGenie. The device has cylindrical body with omni-directional speakers and an LED light ring at the bottom of the device. The Genie provides features like web searches, music streaming, control home automation devices and order products by using Tmall Voice interaction. The device is currently only available in Mandarin ${ }^{[18]}$.

From Russia, according to the survey The Sphinx was an experimental project for a home automation system, commissioned by the Soviet State Committee for Science and Technology and designed by Dmitry Azrikan, in collaboration with A. Kolotushkin and V. Goessen, in 1987. The home environment, as described in a Soviet magazine Technical Aesthetics (Техническаяэстетика in Russian), would be composed of "spherical speakers, a detachable monitor, headphones, a handheld remote control with a removable display, a diskette drive, a processor with three memory blocks and more". The modules were designed to be used by family members, and the number of memory blocks was supposed to be increased endlessly according to the needs of the household, so different family members could control the home devices simultaneously ${ }^{[19]}$.

From India, according to the survey a report published by RedSeer Consulting, Home Automation market in India is rapidly growing and expected to reach INR 8800 crores by 2017. The key growth drivers for this demand are increasing consumer awareness and financial ability, product innovations (like smart phone apps), builders' requirement for market differentiation and an increase for energy conservation efficient systems. There are many players in this field such as Oakter, Inoho, IFIHOMES.com, Silvan Innovation Labs,
eDomotics, SharpNode, Home Brain, MSK Technology, Thasmai Automation and Metagan among others ${ }^{[20]}$.

\section{CONCLUSION}

The IOT conceptualize base smarter, safer and automated house. The concept includes the features like door security, smart car parking which provides a safest security for house. Later there is a water level indicator for storage of water, operating lights, fans \& windows \& also providing a fire alarm system for house.

\section{REFERENCES}

[1] N.H, Ismail, Zarina Tukiran,N.N. Shamsuddin, E.I.S Saadon, "Android-based Home Door Locl(s Application via Bluetooth for Disabled People" Faculty of Electrical and Electronic Engineering Universiti Tun Hussein Onn Malaysia Johor, Malaysia \{nhuda, zarin\} @uthm.edu.my, nurulnadia48@gmail.com, Pusat Pengajian Diploma, Universiti Tun Hussein Onn Malaysia Johor, Malaysia eddy@uthm.edu.my.

[2] Amirah 'Aisha Badrul Hishama, Mohamad Hafis Izran Ishak $^{\mathrm{a} *}$, Chan Kok Teik, Zaharuddin Mohamed Nurul Hawani Idris "Bluetooth-Based Home Automation System Using an Android Phone" Control and Mechatronic Engineering Department, Universiti Teknologi Malaysia, 81310 UTM Johor Bahru, Johor, Malaysia, Faculty of Geoinformation and Real Estate, Universiti Teknologi Malaysia, 81310 UTM Johor Bahru, Johor, Malaysia.

[3] https://www.google.com/url?sa=i\&source=images\&cd= $\&$ cad $=$ rja\&uact $=8 \&$ ved $=2$ ahUKEwjy0oaKg9rkAhXq4X MBHf08Ca0QjRx6BAgBEAQ\&url=\%2Furl\%3Fsa\%3Di $\% 26$ source\%3Dimages\%26cd\%3D\%26ved\%3D\%26url $\% 3 \mathrm{Dhttps} \% 253 \mathrm{~A} \% 252 \mathrm{~F} \% 252 \mathrm{Fpotentiallabs.com} \% 252 \mathrm{~F}$ cart\%252Fbuy-bluetooth-module-online-hyderabadindia\%26psig\%3DAOvVaw1Bjo6p3ZT0LeMG46DJm6P\%26ust\%3D1568884154770129\& psig=AOvVaw1Bjo6p3ZT0LeMG46DJm6P \&ust=15688 84154770129

[4] https://www.google.com/url?sa=i\&source=images\&cd= \&ved=2ahUKEwjylabMgtrkAhWA4HMBHVxPAwQQj Rx6BAgBEAQ\&url=https\%3A\%2F\%2Fwww.makerlabelectronics.com\%2Fproduct\%2Farduino-uno-r3atmega328pch340g\%2F\&psig=AOvVaw1fhBH_Ribvg7 FAbfPy8iHU\&ust $=1568884018241105$

[5] https://www.google.com/url?sa=i\&source=images\&cd= \&ved=2ahUKEwjBpZLAgdrkAhUOS48KHf0yBn0QjRx 6BAgBEAQ\&url=https $\% 3 \mathrm{~A} \% 2 \mathrm{~F} \% 2 \mathrm{Fwww}$.jpinfotech.or g\%2Fandroid-application development\%2F\&psig=AOvVaw3Q1AWtHa69hMehR 1PGG697\&ust=1568883734749363

[6] https://www.google.com/url?sa=i\&source=images\&cd= $\& \mathrm{cad}=$ rja \&uact $=8 \&$ ved $=2$ ahUKEwjAwOSbg9rkAhUb8 XMBHc69BAoQjRx6BAgBEAQ\&url=\%2Furl\%3Fsa\% 3Di\%26source\%3Dimages\%26cd\%3D\%26ved\%3D\%26 url\%3Dhttps\%253A\%252F\%252Fwww.researchgate.net $\% 252$ Ffigure $\% 252$ FSpeech-recognition-system-block diagram-Several-different-techniques-forfeature_fig1_258650613\%26psig\%3DAOvVaw0khB_R n47VTkapYRF3hElr\%26ust\%3D1568884189571783\&p sig=AOvVaw0khB_Rn47VTkapYRF3hElr\&ust=156888 4189571783

[7] https://www.google.com/url?sa=i\&source=images\&cd= \&ved=2ahUKEwj33pD5gtrkAhXC4HMBHV13CZkQjR 
x6BAgBEAQ\&url=https\%3A\%2F\%2Fwww.getit01.com \%2Fp2018020822709693\%2F\&psig=AOvVaw1gP5Mw SFC-gYVFZjazQNcD\&ust=1568884122726584

[8] Salim Jibrin Danbatta Department of Software Engineering Firat University Elazig," Comparison of Zigbee, Z-Wave, Wi-Fi, and Bluetooth Wireless Technologies Used in Home Automation" Turkey salimdambatta@gmail.com Asaf Varol Department of Software Engineering Firat University Elazig, Turkey varol.asaf@gmail.com.

[9] https://www.elprocus.com/what-is-zigbee-technologyarchitecture-and-its-applications/

[10] https://www.google.com/url?sa=i\&source=images\&cd= $\&$ cad $=$ rja\&uact $=8 \&$ ved $=2$ ahUKEwih 8 -

2IgtrkAhV47HMBHfHlABEQjRx6BAgBEAQ\&url=\%2 Furl\%3Fsa $\% 3 \mathrm{Di} \% 26$ source $\% 3$ Dimages $\% 26 \mathrm{~cd} \% 3 \mathrm{D} \% 26$ ved $\% 3 \mathrm{D} \% 26$ url $\% 3 \mathrm{Dhttps} \% 253 \mathrm{~A} \% 252 \mathrm{~F} \% 252 \mathrm{Fwww}$. the -ambient.com\%252Fguides\%252Fzwave-z-wave-smarthome-guide-281\%26psig\%3DAOvVaw1e0PzTMBkSwvcSaxiDPhw\%26ust\%3D1568883882797711\&p sig=AOvVaw1e0PzT

MBkSwvcSaxiDPhw\&ust=1568883882797711

[11] https://www.google.com/url?sa=i\&source=images\&cd= \&ved=2ahUKEwigw7i4gtrkAhUDheYKHQZ3CA0QjRx 6BAgBEAQ\&url=https\%3A\%2F\%2Fhomautomation.or g\%2F $2013 \% 2 \mathrm{~F} 09 \% 2 \mathrm{~F} 29 \% 2 \mathrm{~F}$ why-using-x 10-for-homeautomation\%2F\&psig=AOvVaw2xPK02IgkNcS1qVte7 Lk3R\&ust $=1568883985598024$

[12] https://www.google.com/url?sa=i\&source=images\&cd= \&ved=2ahUKEwirm_ybgtrkAhUL63MBHTT4DIEQjRx 6BAgBEAQ\&url=https $\% 3 \mathrm{~A} \% 2 \mathrm{~F} \% 2 \mathrm{Fwww}$.drivereasy.c om\%2Fknowledge $\% 2$ Fhow-to-fix-windows-10-wifiwont-turnon\%2F\&psig=AOvVaw3YHmXdMOETVXEnIsckqF5\&ust=1568883921342425

[13] https://www.vectorstock.com/royalty-free-vector/smarthome-iot-internet-of-things-control-comfort-vector21900491

[14] Omar Hamdan, Hassan Shanableh, Inas Zaki. A. R. AlAli "Iot-Based Interactive Dual Mode Smart Home
Automation" Senior Member, IEEE, and Tamer Shanableh, Senior Member, IEEE American University of Sharjah, Sharjah, UAE (b00057329, b00057346, g00057594, aali, tshanableh)@aus.edu.

[15] Md. Sadad Mahamud Dept. of Electrical and Electronic Enginnering American Intenational UniversityBangladesh Dhaka, Bangladesh sadad@aiub.edu "Domicile - An IoT Based Smart Home Automation System" Md. Saniat Rahman Zishan Dept. of Electrical and Electronic Enginnering American International UniversityBnagladesh Dhaka, Bangladesh saniat@aiub.edu, Syed Ishmam Ahmad Dept. of Electrical and Electronic Enginnering American International University- Bangladesh Dhaka, Bangladesh ahmed.ishmam07@gmail.com, Ahmed Rezaur Rahman Dept. of Electrical and Electronic Engineering American International UniversityBangladesh Dhaka, Bangladesh rrezaur72@gmail.com, Mehedi Hasan Dept. of Electrical and Elctronic Enginnering American International UniversityBangladesh Dhaka,Bangladesh redoyhasan123@gmail.com, Md.Lutfur Rahman Dept. of Electrical and Electronic Enginnering American International University- Bangladesh Dhaka, Bnagladesh md.lutfurrahmanaiub@gmail.com

[16] sravi wankhade, Shashank Karhade, Pratik Mohite, Kanchan Dhole ${ }^{1}$, Akash Ganvir, Bharti Khedkar SharayuSangekar "home automation system based on iot using cellular device" BE Student, Department of Computer Technology, Rajiv Gandhi College of Engineering and Research, Nagpur, Maharashtra, IndiaAssistant Professor, Department of Computer Technology, Rajiv Gandhi College of Engineering and Research, Nagpur, Maharashtra, India

[17] https://en.wikipedia.org/wiki/Home_automation

[18] https://en.wikipedia.org/wiki/Tmall_Genie

[19] https://en.wikipedia.org/wiki/Sphinx_(home_automation _system)

[20] https://www.indianweb2.com/2016/08/10/10-promisinghome-automation-startups-india/ 